\title{
A Liposomal Platform for Delivery of a Protein Antigen to Langerin- Expressing Cells
}

\author{
Jessica Schulze, ${ }^{\dagger, \ddagger}$ Mareike Rentzsch, ${ }^{\dagger}$ Dongyoon Kim, ${ }^{\dagger}$ Lydia Bellmann, ${ }^{\S}$ Patrizia Stoitzner, ${ }^{\S}$ \\ and Christoph Rademacher*, ${ }^{*},+0$ \\ ${ }^{\dagger}$ Max Planck Institute of Colloids and Interfaces, Department of Biomolecular Systems, 14424 Potsdam, Germany \\ ${ }^{\ddagger}$ Freie Universität Berlin, Department of Biology, Chemistry and Pharmacy, 14195 Berlin, Germany \\ ${ }^{\S}$ Medical University of Innsbruck, Department of Dermatology, Venereology and Allergology, 6020 Innsbruck, Austria
}

Supporting Information

\begin{abstract}
The skin is an attractive site for vaccination and harbors a dense network of Langerhans cells that are the prime target for antigen delivery approaches in the epidermis. While specific targeting of Langerhans cells has been shown to elicit the necessary T-cell response using antibody-based delivery approaches, the targeted administration of particulate antigens in the form of nanoparticle-based vaccine formulations has been challenging. We previously reported on a specific targeting ligand for human Langerin, a C-type lectin expressed on Langerhans cells. This ligand is presented on liposomes and renders them highly specific for the uptake by Langerhans cells. Here we show a detailed study of the uptake and intracellular routing of the particles in model cell lines by

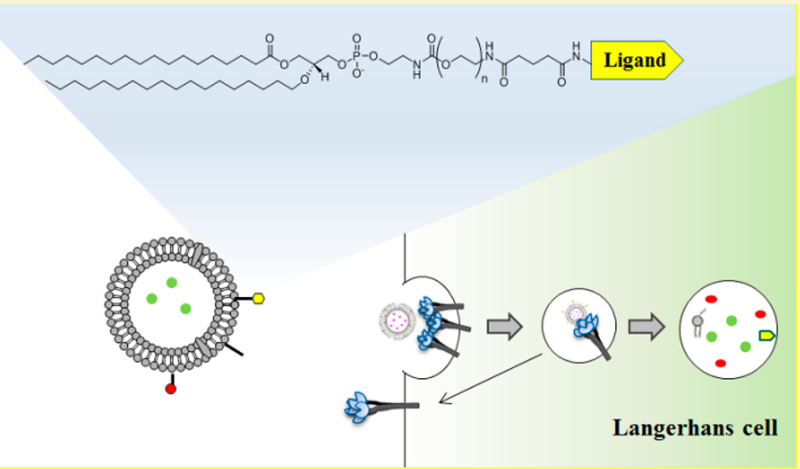
confocal and live cell imaging as well as flow cytometric assays. Liposomes are internalized into early endosomal compartments and accumulate in late endosomes and lysosomes, shortly followed by a release of the cargo. Furthermore, we show the encapsulation of protein antigens and their delivery to cell lines and primary human Langerhans cells. These data further support the applicability of the targeted liposomal particles for protein vaccine applications.
\end{abstract}

A majority of conventional vaccines are applied intramuscularly and subcutaneously. However, only a few antigen-presenting cells reside in these tissues. ${ }^{1}$ In contrast, the skin has a high density of resident immune cells and has been targeted for drug delivery. ${ }^{2}$ In particular, the epidermis provides integrity mainly generated by keratinocytes as well as immune protection via Langerhans cells (LCs). These immune cells make up $1-3 \%$ of all epidermal cells and are the only antigenpresenting cells that function as "gatekeepers" in the epidermis. ${ }^{3}$ LCs are capable of migrating to the skin-draining lymph node where antigens are presented to cells of the adaptive immune system. In a steady state, a small fraction of LCs circulates and thereby induces tolerance. ${ }^{4}$ On the other hand, LCs can activate an immune response against pathogens such as Candida albicans. ${ }^{5}$ Overall, LCs can internalize pathogens, allergens, and cancer antigens for $\mathrm{T}$-cell activation in the draining lymph nodes. ${ }^{3,6}$ Consequently, these immune cells are an attractive target for immunotherapy to improve skin-based vaccination. ${ }^{7}$

A number of studies demonstrated the potential of targeting skin dendritic cells (DCs) with antibodies against specifically expressed cell surface receptors such as DEC-205 and Langerin. $^{8-12}$ Langerin is a C-type lectin receptor (CLR) expressed on Langerhans cells in the human skin and has been a target receptor for eliciting antiviral and antitumor responses (UniProtKB Q9UJ71). ${ }^{10,13-16}$ Engagement of the receptor leads to rapid cargo uptake indicative of receptor-mediated endocytosis. However, a classical internalization motif is missing in its 43-amino acid intracellular domain. ${ }^{13,17}$ Thus far, Langerin has been associated with the actin cytoskeleton via binding of LSP1 to the intracellular domain, which likely provides a means for cargo shuttling. ${ }^{18}$ Nevertheless, clathrin-mediated endocytosis and caveolin-1 positive vesicles have been reported for Langerin, and a lysosomal degradation pathway has been observed. ${ }^{17,19-21}$ Intracellularly, Langerin is preferentially located in pools of the $\mathrm{Rab} 11^{+} / \mathrm{CD} 1 \mathrm{a}^{+}$positive recycling compartment, regulated by its association with the myosin Vb/Rab11A/Rab11-FIP2 platform. ${ }^{17,22,23}$ The cycling between internal pools and extracellular presentation is likely very fast with rates $\left(T_{1 / 2}\right)$ of $22 \mathrm{~s}$ being reported. ${ }^{22}$

Several pathogens, including HIV, measles, influenza A virus, Mycobacterium lepra, Mycobacterium tuberculosis, and Yersinia pestis, ${ }^{21,24-26}$ are recognized by Langerin on the basis of their carbohydrate signature. ${ }^{13,27}$ The cargo is released in the early endosomal compartment from where the receptor recycles back to the surface. ${ }^{13,22}$ This mechanism has been explored for targeted antigen approaches using antibody-based delivery. In

Received: May 3, 2019

Published: May 7, 2019 
mice, HIV antigens have successfully been delivered to Langerin-expressing cells. ${ }^{16}$ These data are further substantiated by delivery of the model antigen ovalbumin (OVA) ${ }^{28}$ Antigen delivery to human LCs mediated by Langerin antibodies to human LCs showed enhanced co-localization with EEA-1, highlighting the potential therapeutic use in humans. ${ }^{29}$

As an alternative to antibody-based delivery, liposomal formulations decorated with natural ligands for Langerin such as Le ${ }^{\mathrm{Y}}$ blood group antigens have been investigated. ${ }^{14}$ Antigens as well as adjuvants can be encapsulated into liposomes harboring great potential for therapeutic applications. The recognition of carbohydrate ligands is mediated by the central cofactor $\mathrm{Ca}^{2+}$, which coordinates two hydroxyl groups of the glycan. This cofactor usage is part of the binding and release mechanism, essential for the efficient pathogen recognition and processing machinery. The $\mathrm{Ca}^{2+}$ concentration and $\mathrm{pH}$ of the extracellular environment are key factors for ligand binding, while changes in these parameters associated with the endosomal compartment result in carrier release. ${ }^{30}$ However, carbohydrate-based delivery vehicles suffer from low specificity because many receptors such as DC-SIGN, dectin-2, and the mannose receptor recognize mannose-type glycans. We recently described the development of a synthetic ligand for human Langerin, an $\mathrm{N}$-tosylated glucosamine derivative, with sufficient affinity and specificity allowing uptake of liposomes into Langerin-expressing model cell lines and primary LCs from human skin. ${ }^{31}$

To induce long-lasting cellular and humoral immune responses against protein antigens, the cargo has to be released in endosomal compartments. Whereas cargo release in early endosomal compartments is associated with MHC class I presentation, late endosomal to lysosomal antigen release mediates MHC class II presentation. ${ }^{32}$ Antigen encapsulation into $\mathrm{pH}$-dependent liposomes can control the delivery of an antigen to defined cellular compartments and therefore direct the immune response to $\mathrm{CD} 4^{+}$or $\mathrm{CD} 8^{+}$T-cell activation. ${ }^{33}$ However, under inflammatory conditions, dendritic cells are capable of producing reactive oxygen species (ROS) that release antigens into the cytosol due to membrane destabilization by lipid peroxidation and thereby cause cross-presentation. ${ }^{34}$

Here, we monitored liposomal routing into Langerinexpressing cell lines by co-formulation with the $\mathrm{pH}$-sensitive dye pHrodo. Moreover, encapsulation of the self-quenching dye calcein allowed detection of antigen release. We further encapsulated a FITC-labeled test protein and presented specific delivery to Langerin-expressing cell lines as well as to primary LCs in epidermal cell suspensions.

Liposomes that were $140 \pm 23 \mathrm{~nm}$ in diameter were formulated using a 57:38:5 DSPC/cholesterol/DSPE-PEG mixture, as either a targeted or a nontargeted formulation, including $4.75 \mathrm{~mol} \%$ DSPE-PEG-GlcNTosyl and $0.25 \mathrm{~mol} \%$ DSPE-PEG-Alexafluor647 (A647). To study the uptake of liposomes into endosomal compartments, the $\mathrm{pH}$-sensitive dye pHrodo red was included in the liposomal formulation. Therefore, a second set of liposomes was formulated containing only $4.5 \mathrm{~mol} \%$ DSPE-PEG-GlcNTosyl as a targeting ligand but utilizing equal amounts $(0.25 \mathrm{~mol} \%)$ of pHrodo and A647 for tracking the uptake and routing of liposomes into Langerinexpressing Hek293 cells. Adherent Hek293 cells were used to stain endosomal markers with antibodies. Liposomes containing only pHrodo or A647 were used as a control (Figure S1). A time series of confocal images shows the uptake of liposomes into cells by accumulation of punctuate A647 fluorescence in endosomal compartments (Figure 1A).

Next, live cell imaging data were recorded to cover a time span of $4 \mathrm{~h}$, monitoring the binding and uptake of the doubly labeled liposomes to Langerin-expressing cells (Figure S2A,B). An

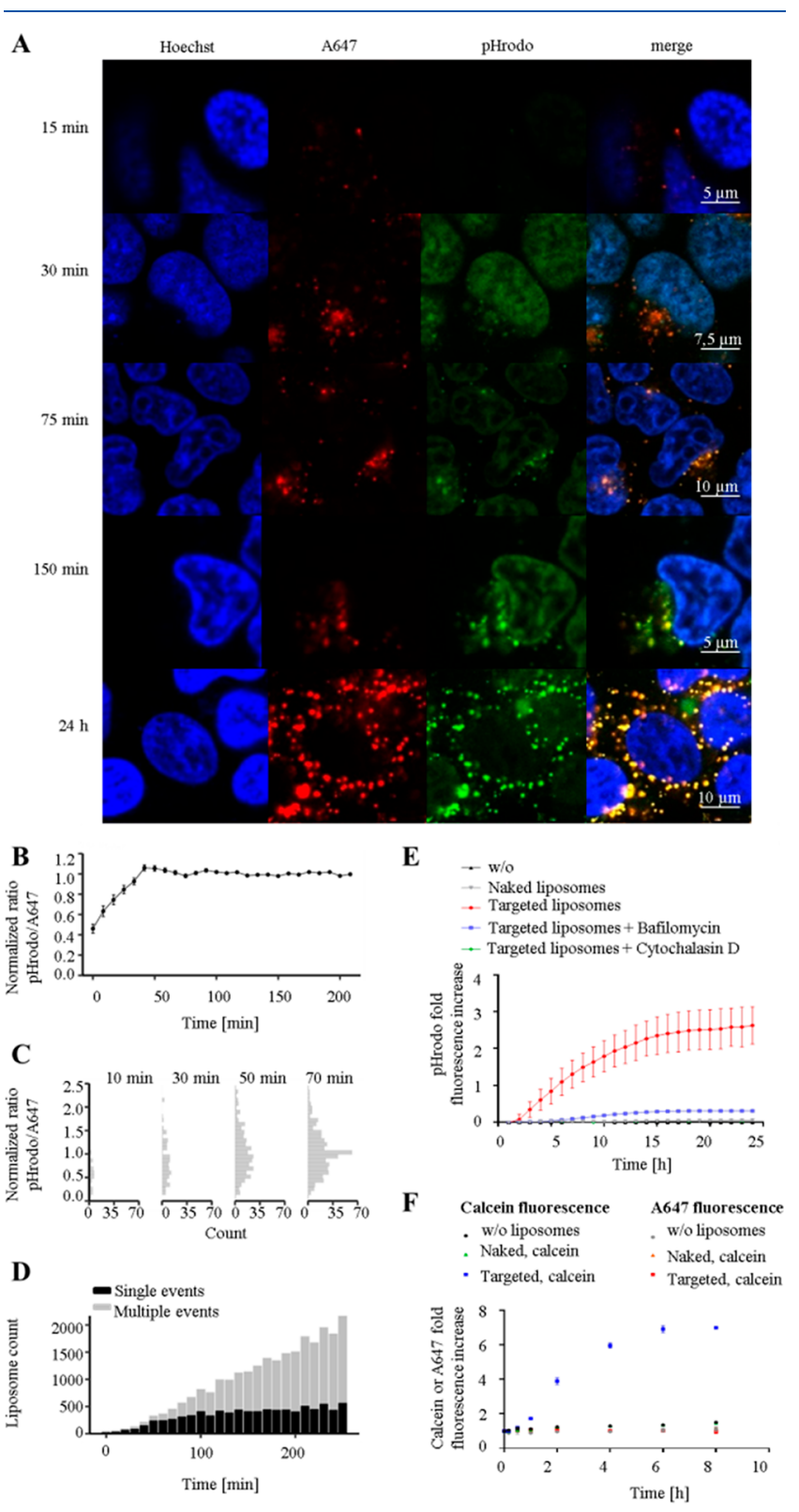

Figure 1. Liposomal routing and cargo release in human Langerin positive cells. (A) pHrodo and A647-labeled liposomes were tracked via confocal microscopy in human Langerin-expressing Hek293 cells at different time points. (B) pHrodo/A647 fluorescence intensity ratio monitored over $4 \mathrm{~h}$ during live cell imaging. (C) Representative histogram of the pHrodo/A647 fluorescence intensity ratio for 10, 30, 50 , and 70 min snapshots. (D) Number of single (black) and multiple liposome events (gray) over time. The error bars represent standard error of the mean. (E) Live cell imaging of the pHrodo fluorescence recorded with a Cytation5 imager. Liposomal internalization was inhibited with $6.25 \mathrm{nM}$ Bafilomycin and $100 \mathrm{nM}$ Cytochalasin D. (F) Time-dependent release of the self-quenching calcein dye from liposomes monitored to assess cargo release kinetics in Langerinexpressing Raji cells. The error bars represent the standard deviation of triplicate measurements from one experiment. 
almost linear increase in the pHrodo/A647 ratio was observed over the first $50 \mathrm{~min}$ followed by a plateau indicating no acidification of the environment of the liposomes at later time points (Figure 1B). The linear increase in the ratio of the two dyes likely results from an accumulation of the liposomes in acidic late endosomal/lysosomal compartments over time. The plateau after 50 min suggests that the majority of liposomes is now located in acidic compartments. Representative histograms of the intensity ratios from four time points highlight the shift in the mean intensity indicating a $\mathrm{pH}$ increase and furthermore show the increased number of liposomes being taken up over time (Figure 1C). This steady increase in the number of internalized liposomes over time during live cell imaging is in line with our findings from confocal imaging (Figure 1A). Moreover, a high level of co-localization with late endosomal/ lysosomal marker Lamp-1 after longer incubation times was confirmed by immunofluorescence, while markers of other endosomal compartments did not show a significant colocalization (Rab7, Rab11, and EEA1) in Hek293 cells after 2 h (Figure S3). Consequently, a low pHrodo/A647 ratio can be associated with early endosomal compartments.

These data suggested a late endosomal/lysosomal accumulation of the liposomes being continuously supplied by newly incorporated liposomes going through the early endosomal compartment. On the basis of the fluorescence intensity of early time point data, we were able to differentiate the occurrence of single from multiple liposomes within a cell (Figure 1D). Single liposomes being taken up into the cells had lower pHrodo/A647 ratios and were smaller in size, suggesting passage through the early endosomal compartment. Higher levels of accumulation of the liposomes had a higher dye ratio and were enriched at later time points. One likely explanation is membrane fusion of early endosomal compartments into late endosomal/lysosomal compartments. While these later stages provide a means for the steady uptake of the liposomes over extended periods of time, early endosomes are quickly saturated after approximately $1 \mathrm{~h}$ and hence correspond to the bottleneck of the uptake process.

Finally, these data about the routing of liposomal vehicles were further supported by inhibitor studies using Bafilomycin, a V-ATPase inhibitor that prevents endosome acidification, and Cytochalasin D, which stops endocytosis by inhibiting actin polymerization (Figure 1E and Figure S4). Over an extended time course of $24 \mathrm{~h}$, both inhibitors strongly reduced the intensity of the fluorescence signal of pHrodo, supporting the findings that liposomes are internalized into late endosomal/ lysosomal compartments, which are associated with antigen loading on MHC class II. ${ }^{32}$

To study the effect of acidic compartments on liposome integrity, liposomes were incubated with buffers of different pHs. Size as well as monodispersity was then analyzed by DLS (Figure S5A). Even after incubation for $48 \mathrm{~h}$ under acidic conditions, fully intact liposomes were detected. To substantiate these findings further, we applied calcein-filled liposomes. Calcein is a self-quenching dye and was incorporated into liposomes at a high concentration. First, its self-quenching effect was detected by dissolving calcein-encapsulated liposomes due to addition of a detergent (Figure S5B). The effect of $\mathrm{pH}$ and calcium on calcein was negligible compared to the selfquenching effect (Figure S5C-E). Consequently, calceinencapsulated liposomes allowed the detection of fluorescence increases when the cargo was released in endosomal compartments. In a pulse chase experiment, liposomes were incubated for $1 \mathrm{~h}$ at $4{ }^{\circ} \mathrm{C}$, washed extensively prior to being warmed, and monitored over a period of $8 \mathrm{~h}$ for the increase in fluorescence by flow cytometry. A steady increase in fluorescence going into saturation at $6 \mathrm{~h}$ was observed (Figure $1 \mathrm{~F}$ ). These data give clear evidence that small molecules encapsulated into liposomes can be delivered to Langerin-expressing cells. Moreover, these data are in line with an uptake into the late endosomal/lysosomal compartment where likely lysosomal acidic lipases are active, leading to cargo release. ${ }^{35}$

To further explore the applicability of the liposomal delivery for the delivery of an antigen to Langerhans cells, we encapsulated a model protein antigen, FITC-labeled bovine serum albumin (BSA). Encapsulation was optimized, and ultracentrifugation demonstrated the best purification method of loaded liposomes from free protein (Figures S6 and S7). Hence, the two labels allowed differentiation between the vehicle and cargo. After incubation of these liposomes with Langerin-expressing Raji cells for $2 \mathrm{~h}$ at $37^{\circ} \mathrm{C}$, a Langerin specific delivery of the FITC-labeled BSA antigen was observed (Figure 2A). Furthermore, these data underscore the high specificity of the targeted liposomes over nontargeted vehicles. Via analysis of a dose- and time-dependent uptake, a high correlation of vehicle and cargo was detected, indicating a similar location in cellular compartments (Figure 2B,C). Finally, the uptake of these antigen-filled liposomes was monitored using

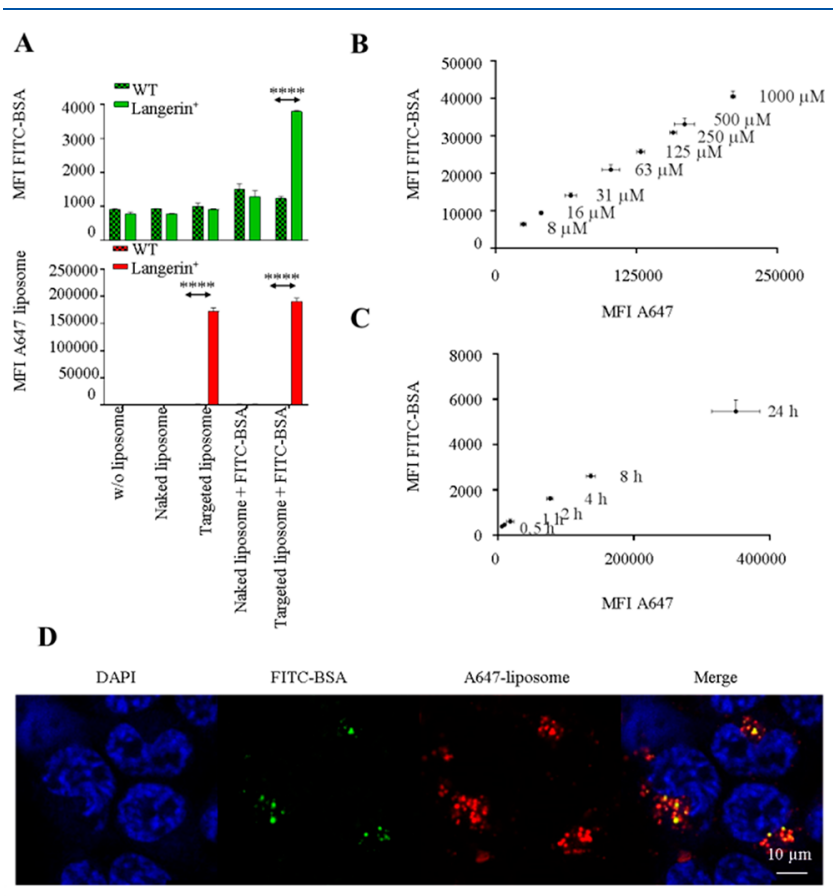

Figure 2. In vitro delivery of an antigen to Langerin-expressing cell lines. (A) FITC-BSA-encapsulated liposomes were used in a cell-based assay. Liposomes were incubated for $2 \mathrm{~h}$ at $37{ }^{\circ} \mathrm{C}$, and FITC and A647 fluorescence were measured by flow cytometry $(* * * * p<0.0001 ; n=3$; two-tailed, unpaired Student's $t$ test; one of three representative experiments). Comparison of A647 (lipid-conjugated) and FITC (cargo-conjugated) fluorescence in a (B) dose-dependent and (C) time-dependent manner. The error bars represent the standard deviation from one representative experiment with $n=3$ of at least two independent experiments. (D) FITC-BSA-encapsulated liposomes were incubated with Langerin-expressing Hek293 cells for $6 \mathrm{~h}$ at $37^{\circ} \mathrm{C}$. The nucleus was stained with DAPI, and cells were analyzed by confocal microscopy. 
confocal imaging supporting these previous findings (Figure 2D).

Thus far, our data strongly support efficient and specific delivery into Langerin-expressing cell lines (Raji and Hek293). To expand our data to primary cells, we made use of epidermal cell suspensions and the therein contained $1-3 \%$ of LCs. These cell suspensions were exposed to targeted and nontargeted liposomes for $2 \mathrm{~h}$ (Figure 3). While the latter did not bind to any

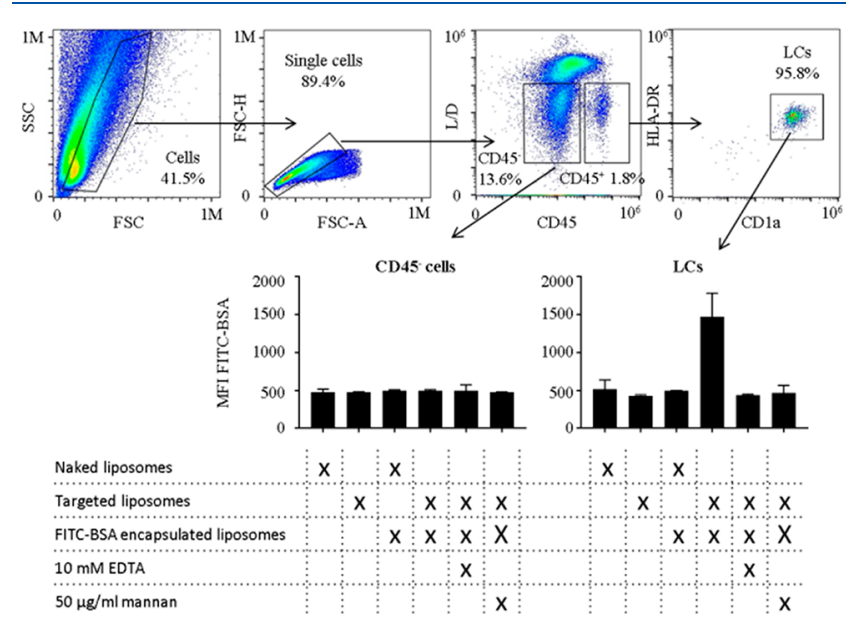

Figure 3. In vitro delivery of an antigen to human LCs in epidermal cell suspensions. FITC-BSA-encapsulated liposomes were incubated with epidermal cell suspensions for $2 \mathrm{~h}$ at $37^{\circ} \mathrm{C}$. LCs were identified as viable CD $45^{+} \mathrm{CD} 1 \mathrm{a}^{+} \mathrm{HLA}-\mathrm{DR}^{+}$cells. The MFI of FITC-BSA was plotted for CD45 ${ }^{-}$cells and LCs. Liposomes devoid of the targeting ligand (naked liposomes), EDTA to sequester the essential cofactor $\mathrm{Ca}^{2+}$ for ligand recognition by Langerin, and the polysaccharide mannan served as controls. Error bars represent the standard deviation of triplicate measurements from one representative experiment of two independent ones.

of the cells, targeted liposomes encapsulated with FITC-BSA were detected in LCs characterized as viable $\mathrm{CD} 45^{+} \mathrm{CD} 1 \mathrm{a}^{+} \mathrm{HLA}-\mathrm{DR}^{+}$cells. $\mathrm{CD}^{-} 5^{-}$cells, such as keratinocytes, did not bind the liposomes. Additional controls were employed to show specificity. First, EDTA was able to abrogate the $\mathrm{Ca}^{2+}$-dependent recognition of the targeting ligand, and second, mannan, a mannose-containing polysaccharide from Saccharomyces cerevisiae, also resulted in complete inhibition of the liposomal uptake into LCs, confirming binding to the primary binding site.

Overall, we could further support previous findings that our targeting ligand is specific for Langerin-expressing cells and that the liposomal delivery platform can be used to deliver small molecules as well as encapsulated proteins to model cell lines and primary cells. Hence, the fact that the epidermis is an easily accessible vaccination site and that Langerin has a restricted expression profile on LCs renders the liposomal delivery platform an attractive tool for novel therapeutic applications.

\section{ASSOCIATED CONTENT}

\section{S Supporting Information}

The Supporting Information is available free of charge on the ACS Publications website at DOI: 10.1021/acs.biochem.9b00402.

Detailed material and method information and Figures S1-S7 (PDF)

\section{Accession Codes}

Langerin, Q9UJ71.

\section{AUTHOR INFORMATION}

\section{Corresponding Author}

*E-mail: christoph.rademacher@mpikg.mpg.de.

ORCID $\odot$

Christoph Rademacher: 0000-0001-7082-7239

\section{Funding}

The work was supported by the DFG (RA1944/2-1 and RA1944/6-1), the European Research Council (ERC; ERC Starting Grant 716024), and the Max Planck Society.

\section{Notes}

The authors declare the following competing financial interest(s): J.S. and C.R. declare the filing of a patent covering the use of glycomimetic Langerin ligands for targeting human Langerin-expressing cells. Internationale Patentanmeldung PCT/EP2019/051055.

\section{ACKNOWLEDGMENTS}

The authors thank Prof. Dr. Peter H. Seeberger for support and helpful discussions.

\section{ABBREVIATIONS}

A647, Alexafluor647; BSA, bovine serum albumin; $\mathrm{Ca}^{2+}$, calcium; CLR, C-type lectin receptor; DC-SIGN, dendritic cell specific intercellular adhesion molecule-3 grabbing nonintegrin; DLS, dynamic light scattering; DSPC, 1,2-distearoyl$s n$-glycero-3-phosphocholine; DSPE, 1,2-distearoyl-sn-glycero3-phosphoethanolamine; EDTA, ethylenediaminetetraacetic acid; EEA1, early endosome antigen 1; FITC, fluorescein isothiocyanate; HIV, human immunodeficiency virus; Lamp1, lysosomal-associated membrane protein 1; LC, Langerhans cell; LSP1, lymphocyte specific protein 1; MHC, major histocompatibility molecule; OVA, ovalbumin; PEG, polyethylene glycol; ROS, reactive oxygen species; $T_{1 / 2}$, half-life.

\section{REFERENCES}

(1) Combadiere, B., and Liard, C. (2011) Transcutaneous and intradermal vaccination. Hum. Vaccines 7, 811-827.

(2) Prausnitz, M. R., and Langer, R. (2008) Transdermal drug delivery. Nat. Biotechnol. 26, 1261-1268.

(3) Romani, N., Clausen, B. E., and Stoitzner, P. (2010) Langerhans cells and more: langerin-expressing dendritic cell subsets in the skin. Immunol. Rev. 234, 120-141.

(4) Kaplan, D. H. (2017) Ontogeny and function of murine epidermal Langerhans cells. Nat. Immunol. 18, 1068-1075.

(5) Haley, K., Igyarto, B. Z., Ortner, D., Bobr, A., Kashem, S., Schenten, D., and Kaplan, D. H. (2012) Langerhans Cells Require MyD88-Dependent Signals for Candida albicans Response but Not for Contact Hypersensitivity or Migration. J. Immunol. 188, 4334-4339.

(6) Clausen, B. E., and Stoitzner, P. (2015) Functional Specialization of Skin Dendritic Cell Subsets in Regulating T Cell Responses. Front. Immunol. 6, 534.

(7) Stoitzner, P., Sparber, F., and Tripp, C. H. (2010) Langerhans cells as targets for immunotherapy against skin cancer. Immunol. Cell Biol. 88, $431-437$.

(8) Flacher, V., Tripp, C. H., Stoitzner, P., Haid, B., Ebner, S., Del Frari, B., Koch, F., Park, C. G., Steinman, R. M., Idoyaga, J., and Romani, N. (2010) Epidermal Langerhans cells rapidly capture and present antigens from C-type lectin-targeting antibodies deposited in the dermis. J. Invest. Dermatol. 130, 755-762.

(9) Flacher, V., Tripp, C. H., Haid, B., Kissenpfennig, A., Malissen, B., Stoitzner, P., Idoyaga, J., and Romani, N. (2012) Skin langerin+ 
dendritic cells transport intradermally injected anti-DEC-205 antibodies but are not essential for subsequent cytotoxic CD8+ $\mathrm{T}$ cell responses. J. Immunol. 188, 2146-2155.

(10) Flacher, V., Tripp, C. H., Mairhofer, D. G., Steinman, R. M., Stoitzner, P., Idoyaga, J., and Romani, N. (2014) Murine Langerin+ dermal dendritic cells prime CD8+ $\mathrm{T}$ cells while Langerhans cells induce cross-tolerance. EMBO Mol. Med. 6, 1191-1204.

(11) Idoyaga, J., Fiorese, C., Zbytnuik, L., Lubkin, A., Miller, J., Malissen, B., Mucida, D., Merad, M., and Steinman, R. M. (2013) Specialized role of migratory dendritic cells in peripheral tolerance induction. J. Clin. Invest. 123, 844-854.

(12) Stoitzner, P., Schaffenrath, S., Tripp, C. H., Reider, D., Komenda, K., Del Frari, B., Djedovic, G., Ebner, S., and Romani, N. (2014) Human skin dendritic cells can be targeted in situ by intradermal injection of antibodies against lectin receptors. Exp Dermatol. 23, 909915.

(13) Valladeau, J., Ravel, O., Dezutter-Dambuyant, C., Moore, K., Kleijmeer, M., Liu, Y., Duvert-Frances, V., Vincent, C., Schmitt, D., Davoust, J., Caux, C., Lebecque, S., and Saeland, S. (2000) Langerin, a novel C-type lectin specific to Langerhans cells, is an endocytic receptor that induces the formation of Birbeck granules. Immunity 12, 71-81.

(14) Fehres, C. M., Kalay, H., Bruijns, S. C., Musaafir, S. A., Ambrosini, M., Van Bloois, L., Van Vliet, S. J., Storm, G., Garcia-Vallejo, J. J., and Van Kooyk, Y. (2015) Cross-presentation through langerin and DCSIGN targeting requires different formulations of glycan-modified antigens. J. Controlled Release 203, 67-76.

(15) Fehres, C. M., Duinkerken, S., Bruijns, S. C., Kalay, H., Van Vliet, S. J., Ambrosini, M., De Gruijl, T. D., Unger, W. W., Garcia-Vallejo, J. J., and Van Kooyk, Y. (2017) Langerin-mediated internalization of a modified peptide routes antigens to early endosomes and enhances cross-presentation by human Langerhans cells, Cellular \&. Cell. Mol. Immunol. 14, 360-370.

(16) Idoyaga, J., Lubkin, A., Fiorese, C., Lahoud, M. H., Caminschi, I., Huang, Y., Rodriguez, A., Clausen, B. E., Park, C. G., Trumpfheller, C., and Steinman, R. M. (2011) Comparable T helper 1 (Th1) and CD8 Tcell immunity by targeting HIV gag p24 to CD8 dendritic cells within antibodies to Langerin, DEC205, and Clec9A. Proc. Natl. Acad. Sci. U.S. A. 108, 2384-2389.

(17) Mc Dermott, R., Ziylan, U., Spehner, D., Bausinger, H., Lipsker, D., Mommaas, M., Cazenave, J. P., Raposo, G., Goud, B., De la Salle, H., Salamero, J., and Hanau, D. (2002) Birbeck granules are subdomains of endosomal recycling compartment in human epidermal Langerhans cells. Mol. Biol. Cell 13, 317-335.

(18) Ribeiro, C. M. S., Sarrami-Forooshani, R., Setiawan, L. C., Zijlstra-Willems, E. M., Van Hamme, J. L., Tigchelaar, W., Van der Wel, N. N., Kootstra, N. A., Gringhuis, S. I., and Geijtenbeek, T. B. H. (2016) Receptor usage dictates HIV-1 restriction by human TRIM5 alpha in dendritic cell subsets. Nature 540, 448-452.

(19) Van den Berg, L. M., Ribeiro, C. M. S., Zijlstra-Willems, E. M., De Witte, L., Fluitsma, D., Tigchelaar, W., Everts, V., and Geijtenbeek, T. B. H. (2014) Caveolin-1 mediated uptake via langerin restricts HIV-1 infection in human Langerhans cells. Retrovirology 11, 123.

(20) Van der Vlist, M., De Witte, L., De Vries, R. D., Litjens, M., De Jong, M. A., Fluitsma, D., De Swart, R. L., and Geijtenbeek, T. B. (2011) Human Langerhans cells capture measles virus through Langerin and present viral antigens to $\mathrm{CD} 4(+) \mathrm{T}$ cells but are incapable of crosspresentation. Eur. J. Immunol. 41, 2619-2631.

(21) Ng, W. C., Londrigan, S. L., Nasr, N., Cunningham, A. L., Turville, S., Brooks, A. G., and Reading, P. C. (2016) The C-type Lectin Langerin Functions as a Receptor for Attachment and Infectious Entry of Influenza A Virus. J. Virol. 90, 206-221.

(22) Gidon, A., Bardin, S., Cinquin, B., Boulanger, J., Waharte, F., Heliot, L., De la Salle, H., Hanau, D., Kervrann, C., Goud, B., and Salamero, J. (2012) A Rab11A/myosin Vb/Rab11-FIP2 complex frames two late recycling steps of langerin from the ERC to the plasma membrane. Traffic 13, 815-833.

(23) McDermott, R., Bausinger, H., Fricker, D., Spehner, D., Proamer, F., Lipsker, D., Cazenave, J. P., Goud, B., De La Salle, H., Salamero, J., and Hanau, D. (2004) Reproduction of Langerin/CD207 traffic and
Birbeck granule formation in a human cell line model. J. Invest. Dermatol. 123, 72-77.

(24) Yang, K., Park, C. G., Cheong, C., Bulgheresi, S., Zhang, S., Zhang, P., He, Y., Jiang, L., Huang, H., Ding, H., Wu, Y., Wang, S., Zhang, L., Li, A., Xia, L., Bartra, S. S., Plano, G. V., Skurnik, M., Klena, J. D., and Chen, T. (2015) Host Langerin (CD207) is a receptor for Yersinia pestis phagocytosis and promotes dissemination. Immunol. Cell Biol. 93, 815-824.

(25) De Witte, L., Nabatov, A., Pion, M., Fluitsma, D., De Jong, M. A., De Gruijl, T., Piguet, V., Van Kooyk, Y., and Geijtenbeek, T. B. (2007) Langerin is a natural barrier to HIV-1 transmission by Langerhans cells. Nat. Med. 13, 367-371.

(26) Hunger, R. E., Sieling, P. A., Ochoa, M. T., Sugaya, M., Burdick, A. E., Rea, T. H., Brennan, P. J., Belisle, J. T., Blauvelt, A., Porcelli, S. A., and Modlin, R. L. (2004) Langerhans cells utilize CD1a and langerin to efficiently present nonpeptide antigens to T cells. J. Clin. Invest. 113, 701-708.

(27) Hanske, J., Schulze, J., Aretz, J., McBride, R., Loll, B., Schmidt, H., Knirel, Y., Rabsch, W., Wahl, M. C., Paulson, J. C., and Rademacher, C. (2017) Bacterial Polysaccharide Specificity of the Pattern Recognition Receptor Langerin Is Highly Species-dependent. J. Biol. Chem. 292, $862-871$.

(28) Idoyaga, J., Cheong, C., Suda, K., Suda, N., Kim, J. Y., Lee, H., Park, C. G., and Steinman, R. M. (2008) Cutting edge: langerin/CD207 receptor on dendritic cells mediates efficient antigen presentation on MHC I and II products in vivo. J. Immunol. 180, 3647-3650.

(29) Fehres, C. M., Duinkerken, S., Bruijns, S. C., Kalay, H., Van Vliet, S. J., Ambrosini, M., De Gruijl, T. D., Unger, W. W., Garcia-Vallejo, J. J., and Van Kooyk, Y. (2017) Langerin-mediated internalization of a modified peptide routes antigens to early endosomes and enhances cross-presentation by human Langerhans cells. Cell. Mol. Immunol. 14, $360-370$.

(30) Hanske, J., Aleksic, S., Ballaschk, M., Jurk, M., Shanina, E., Beerbaum, M., Schmieder, P., Keller, B. G., and Rademacher, C. (2016) Intradomain Allosteric Network Modulates Calcium Affinity of the CType Lectin Receptor Langerin. J. Am. Chem. Soc. 138, 12176-12186.

(31) Wamhoff, E. C. S. J., Bellmann, L., Bachem, G., Fuchsberger, F. F., Rademacher, J., Hermann, M., Del Frari, B., Van Dalen, R., Hartmann, D., Van Sorge, N., Seitz, O., Stoitzner, P., and Rademacher, C. A specific, glycomimetic Langerin ligand for human Langerhans cell targeting. ACS Cent. Sci, DOI: 10.1021/acscentsci.9b00093, just accepted.

(32) Germain, R. N. (1994) MHC-dependent antigen processing and peptide presentation: providing ligands for $\mathrm{T}$ lymphocyte activation. Cell 76, 287-299.

(33) Harding, C. V., Collins, D. S., Slot, J. W., Geuze, H. J., and Unanue, E. R. (1991) Liposome-encapsulated antigens are processed in lysosomes, recycled, and presented to T cells. Cell 64, 393-401.

(34) Dingjan, I., Verboogen, D. R., Paardekooper, L. M., Revelo, N. H., Sittig, S. P., Visser, L. J., Mollard, G. F., Henriet, S. S., Figdor, C. G., Ter Beest, M., and Van den Bogaart, G. (2016) Lipid peroxidation causes endosomal antigen release for cross-presentation. Sci. Rep. 6, 22064.

(35) Settembre, C., Fraldi, A., Medina, D. L., and Ballabio, A. (2013) Signals from the lysosome: a control centre for cellular clearance and energy metabolism. Nat. Rev. Mol. Cell Biol. 14, 283-296. 